\title{
Imaging features of intrahepatic bile duct adenoma in MRI
}

\author{
Jufeng Qin ${ }^{1}$, Guofeng Zhou ${ }^{2}$, Xiaohui Sheng ${ }^{1}$, Beidi Liu ${ }^{1}$ \\ ${ }^{1}$ Department of imaging, Affiliated Hospital of Nantong University, Nantong 226001, China; ${ }^{2}$ Department of Radiology, Zhongshan Hospital, \\ Fudan University, Shanghai 200032, China \\ Contributions: (I) Conception and design: J Qin; (II) Administrative support: G Zhou; (III) Provision of study materials or patients: J Qin, G Zhou; (IV) \\ Collection and assembly of data: J Qin, G Zhou; (V) Data analysis and interpretation: All authors; (VI) Manuscript writing: All authors; (VII) Final \\ approval of manuscript: All authors. \\ Correspondence to: Dr. Jufeng Qin. Department of Imaging, Affiliated Hospital of Nantong University, 20 Xishi Road, Nantong 226001, China. \\ Email: qjf283@163.com.
}

Background: Intrahepatic bile duct adenoma (BDA) is a rare benign epithelial hepatic tumor, and is very easy to miss or misdiagnose. This study was to investigate the magnetic resonance imaging (MRI) findings of intrahepatic BDA and to improve the imaging understanding of the disease.

Methods: The clinical and MRI imaging data of 25 cases of intrahepatic BDA confirmed by operation and pathology were analyzed retrospectively from the aspects of tumor location, size and shape, signal characteristics and enhancement mode.

Results: Among the 25 patients, 24 cases (96\%) were single lesions, only 1 case had multiple lesions (3 lesions), a total of 27 lesions. Twelve lesions were located in the left lobe of the liver, 15 lesions were located in the right lobe of the liver, and the lesions were located under or near the hepatic capsule. The lesions were round and the size was 4 to $20 \mathrm{~mm}$, with an average of $10.2 \mathrm{~mm}$. In T1 weighted image (T1WI) sequence, all lesions were low or slightly low signal. In T2 weighted image (T2WI) sequence, 20 lesions were high or slightly high signal (74.1\%), 4 were central equal signal peripheral ring high signal, and 3 were equal signal. In diffusion-weighted imaging (DWI) sequence, 23 lesions were high or slightly high signals (85.2\%), and 4 were ring high signals. Nineteen lesions (70.4\%) showed marked enhancement in the arterial phase, and the portal vein and lag phase continued to strengthen with a slightly higher or equal signal, 6 lesions (22.2\%) showed mild or obvious peripheral ring enhancement in the arterial phase, and the portal vein and lag phase continued to strengthen with a slightly higher signal around the circumference and a slightly lower signal at the center. Eight cases were examined by gadolinium ethoxybenzyl diethylenetriaminepentaacetic acid (GdEOB-DTPA)-enhanced MRI, 7 cases were low signal in hepatobiliary phase, and 1 case was Peripheral low signal.

Conclusions: The MRI findings of intrahepatic BDA have certain characteristics. Careful observation and analysis are helpful to the understanding and diagnosis of the disease.

Keywords: Liver; bile duct adenoma (BDA); magnetic resonance imaging (MRI)

Submitted Dec 10, 2019. Accepted for publication Jan 31, 2020.

doi: $10.21037 /$ tcr.2020.02.19

View this article at: http://dx.doi.org/10.21037/tcr.2020.02.19

\section{Introduction}

Intrahepatic bile duct adenoma (BDA) is a rare benign epithelial hepatic tumor that occurs in the liver, accounting for approximately $1.3 \%$ of primary liver tumors $(1,2)$. Because patients usually have no clinical symptoms and are often accompanied by other lesions inside and outside the liver, the lesions are small, and various imaging examinations are not easy to display or obscure, so it is very easy to miss or misdiagnose. BDA may be difficult to distinguish from metastatic carcinomas, particularly welldifferentiated pancreatic ductal adenocarcinoma (3-6). At 
present, there are few domestic and foreign literatures, especially in the field of imaging, and most of them are case reports. We collected 25 cases of BDA and analyzed their MRI signs to improve the understanding of the disease.

\section{Methods}

\section{General information}

The clinical and MRI images of 25 cases of intrahepatic BDA confirmed by surgery and pathology from December 2015 to June 2019 in Zhongshan Hospital of Fudan University were collected. The study was approved by ethics board of Zhongshan Hospital, Fudan University (No. Y2018-193). There were 13 males and 12 females, aged 2774 years, with an average age of 57.3 years; 17 patients were associated with hepatitis and hepatocellular carcinoma, and 1 patient was associated with intrahepatic bile duct cancer. The remaining 7 patients had no obvious clinical symptoms or were accompanied by extrahepatic lesions. Twenty-five cases were surgically resected. Postoperative pathology and immunohistochemistry confirmed BDA, 4 of which were locally cancerous.

\section{Inspection methods}

Seventeen out of 25 patients underwent plain MRI scanning and dynamic enhanced scanning, using phased array body coils. Plain scanning sequence includes fast spin echo T2WI, TR 3,000-3,500 ms, TE $84 \mathrm{~ms}$; GRE T1WI sequence, TR 110-130 ms, TE $4.8 \mathrm{~ms}$. DWI single shot echo planar imaging (SS-EPI) sequence, TR 2,400 2,600 ms, TE $66 \mathrm{~ms}$, b-values $0,50,500 \mathrm{~mm}^{2} / \mathrm{s}$. Positive and negative phase $\mathrm{T} 1$ spoiled gradient echo axial image. Three-dimensional volumetric interpolated breath-hold examination (3D-VIBE) scanning was used for dynamic enhanced front and rear scanning, TR $5.04 \mathrm{~ms}$, TE $2.31 \mathrm{~ms}$. The contrast agent was Magen vein, with a total volume of $25-30 \mathrm{~mL}$. It was injected through the elbow vein at a flow rate of $2 \mathrm{~mL} / \mathrm{s}$. The arterial phase, portal vein phase and delayed phase were scanned 25-30 s, 70-90 s, and 3 min after contrast injection. Gd-EOB-DTPA enhanced scanning was used in 8 cases.

\section{Observation index}

The tumor location, size, shape, signal characteristics and enhancement methods were observed and analyzed.

\section{Results}

Of the 25 patients, 24 (96\%) had a single lesion and only one had multiple (three) lesions, a total of 27 lesions. Among them, 12 lesions were located in the left lobe of the liver and 15 lesions were located in the right lobe of the liver. Except for 1 lesion which was about $4.5 \mathrm{~cm}$ away from the liver capsule, the other lesions were located under or near the liver capsule $(0-16 \mathrm{~mm}$, average $4.1 \mathrm{~mm})$.

Lesions are round, $4-20 \mathrm{~mm}$ in size, with an average of $10.2 \mathrm{~mm}$, with clear edges. The T1WI sequences were all low or slightly low signals, and there was no change in the positive and negative phase signals. Twenty lesions (74.1\%) in the T2WI sequence were high or slightly high signals. Four lesions were circular high signals around the central equal signal, and three lesions were equal signals. In the DWI sequence, 23 lesions were high or slightly high $(85.2 \%)$, and 4 lesions were circular high signals. 12 lesions were low or slightly low signal in ADC map. Five lesions were high signal (1 of which was ring high signal), and the remaining 10 lesions were unclear. Enhanced examination of 19 lesions (70.4\%) showed obviously strengthened in the arterial phase and the portal phase and delayed phase continued to show a slightly higher or equal signal (Figure 1). Six lesions $(22.2 \%)$ showed mild or obvious peripheral enhancement in the arterial phase, and continuous enhancement in the portal and delayed phases showed a slightly higher peripheral signal and a lower central signal (Figure 2). The two lesions showed mild or obvious enhancement in the arterial phase, and showed low or slightly low signals in the portal and delayed phases. Eight patients underwent enhanced Gd-EOB-DTPA examination. Seven patients in the hepatobiliary specific period were lowsignal (Figure 3), and one was a low-signal peripheral signal.

\section{Discussion}

$\mathrm{BDA}$ is a rare proliferative lesion originating from damaged intrahepatic bile duct epithelium (7). Microscopically, BDAs consist of hyperplastic small bile ducts with non-open lumens, which are not connected to the intralobular bile ducts, with varying degrees of inflammation and fibrosis. As the disease progresses, the number of small bile ducts and inflammatory cells in the lesion decreases, and fibrous tissue increases. Immunohistochemically, CK19, CK7, CD56 and CD10 are positive in the tumor cells (1).

According to an analysis of 152 cases of BDA by Allaire et al. in 1988 (the study has the largest number of cases at 

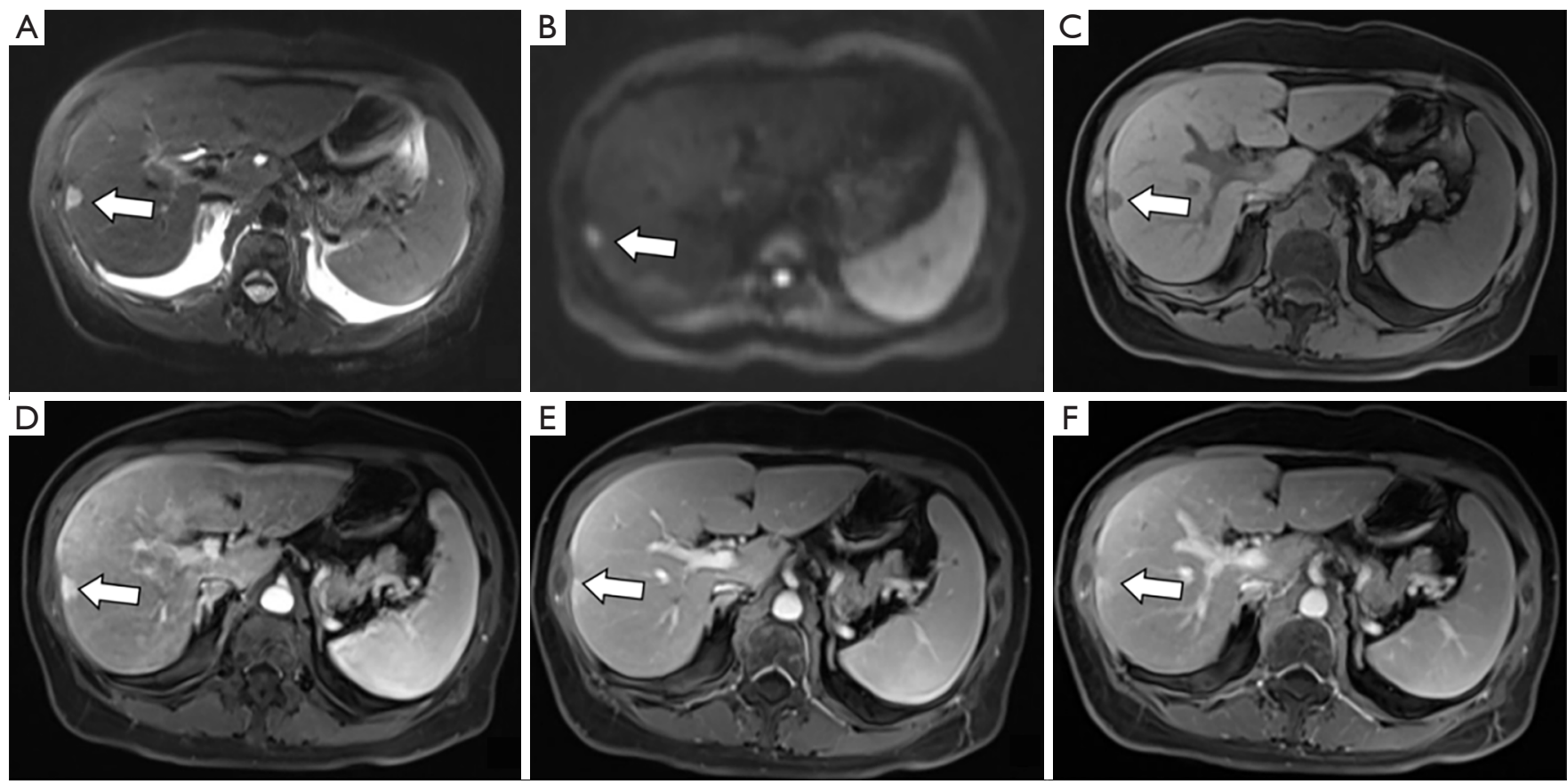

Figure 1 Female, 71 years old, $12 \mathrm{~mm}$ nodules under the right posterior lobe of the liver, high signals in T2WI (A, white arrow) and DWI (B), low signals in T1WI (C), significantly enhanced arterial phase (D), continuous enhancement of portal (E) and delayed phases (F) showed slightly higher signals.
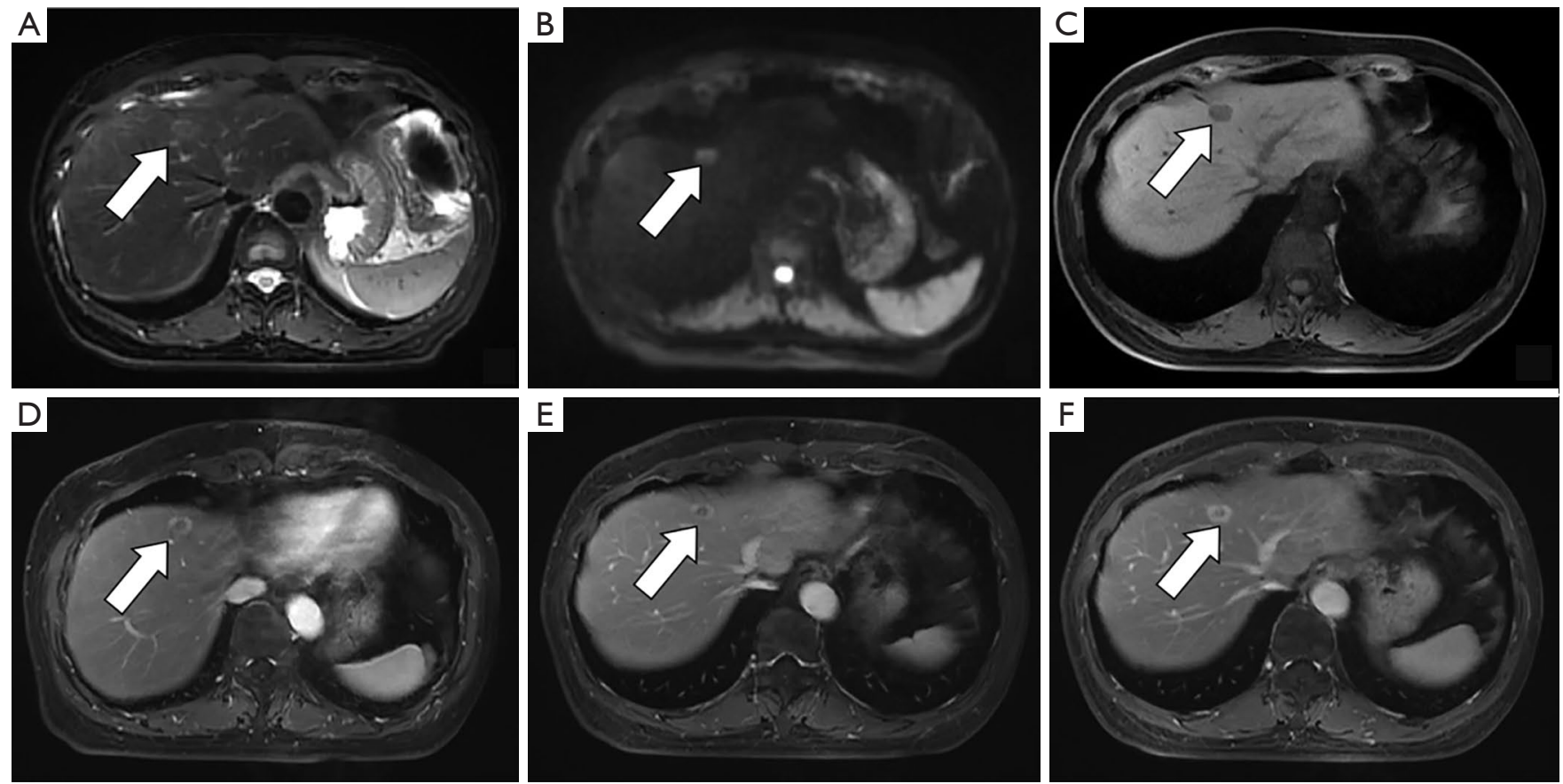

Figure 2 Female, 63 years old, with a $14 \mathrm{~mm}$ nodule near the capsular end of the left inner lobe of the liver. T2WI (A, white arrow) and DWI (B) are slightly high signals, T1WI is low signals (C), and the peripheral phase of the arterial phase is obviously strengthened (D). The continuous enhancement of the portal phase (E) and the delay phase (F) shows a peripheral high signal. 

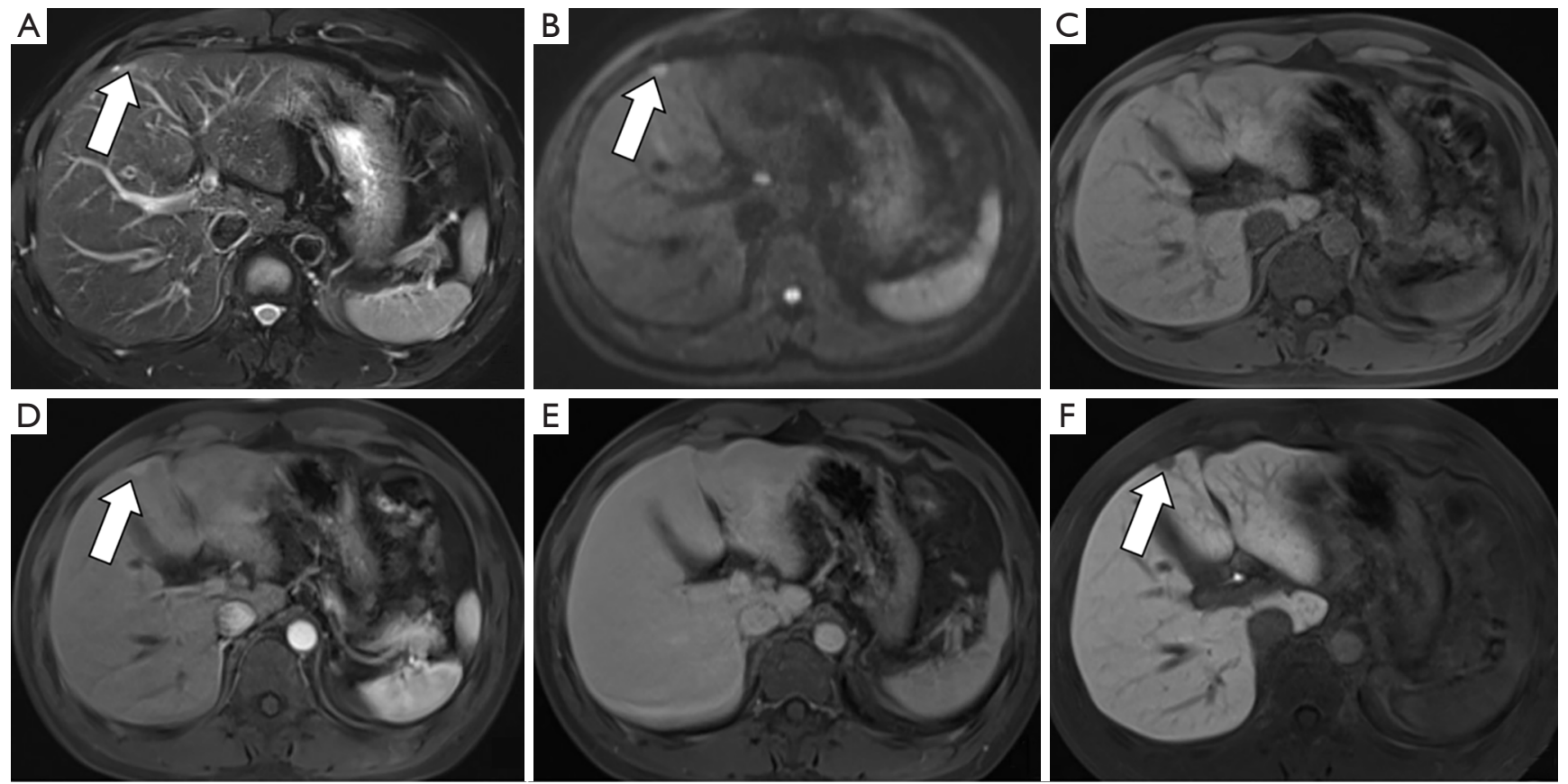

Figure 3 Male, 39 years old, $4 \mathrm{~mm}$ small nodules under the left inner lobe of the liver, with slightly higher T2WI signal (A, white arrow), slightly higher DWI signal (B), lower T1WI signal (C), significantly enhanced arterial phase (D), the delay period showing equal signal (E), hepatobiliary specific phase showing low signal (F).

present), the disease was more common in the age of 20 70 years, the average age is 55 years, and there is no obvious gender difference in incidence (8). In this group of cases, there were 13 males and 12 females. The ratio of male to female was about 1.1:1, and the age was $27-74$ years. The average age was 57.3 years, which was consistent with it. BDA lesions are reported on the surface of the liver or under the capsule (8-11). Twenty-six of the 27 lesions in this group were located under or close to the liver capsule $(0-16 \mathrm{~mm}$ from the liver capsule, average $4.1 \mathrm{~mm}$ ). BDA lesions are generally small, with a diameter of $1-20 \mathrm{~mm}$ and an average of $5.8 \mathrm{~m}$ (8). The size of lesions in this group were $4-20 \mathrm{~mm}$, with an average of $10.2 \mathrm{~mm}$, which was larger than that because we enquired 118 cases of BDA, but 93 of them had too small lesions, which were unclear on MRI or were indistinguishable from other liver lesions. Because of this, it was removed from the group, and most of these 93 lesions were $1-3 \mathrm{~mm}$ in diameter, so the average size of the lesions in this group increased. The largest lesion reported by domestic and foreign literature is $9.2 \mathrm{~cm}$ in diameter (12). Patients usually have no obvious clinical symptoms and signs, no obvious abnormalities in biochemical examination and tumor indicators. It is often found by accident during physical examination, surgery of other liver lesions or surgery of other abdominal organs and autopsy. Although BDA is a benign lesion, it may be malignant, and local resection may be performed if necessary. There were 4 cases of local cancer in this group.

\section{MRI performance}

Based on the analysis of this group of cases and combined with previous literature reports, we believe that the MRI manifestations of BDA are as follows: (I) lesions are located under or near the liver capsule, most of which are $<10 \mathrm{~mm}$ in diameter, and the lesions are usually unclear when the lesion is too small $(1-3 \mathrm{~mm})$. (II) $\mathrm{T} 1 \mathrm{~W}$ is a low or slightly low signal, and the positive and negative phase signals have no change; T2WI is a high or slightly high signal, part of a ring high signal, and very few signals are equal or even slightly lower, which is related to the content of fibrous tissue in the lesion and whether it contains calcification (13); DWI is high or slightly high. (III) There are two main ways to enhance the inspection: (i) the arterial phase was significantly strengthened, and the continuous enhancement of the portal and delayed phases showed a slightly higher 
or equal signal. The characteristic of this continuous enhancement was related to the presence of more fibrous tissue in the lesion (10); (ii) the arterial phase has mild or obvious peripheral ring enhancement, and the portal and delay phases continue to strengthen with a peripheral ring slightly higher signal and a central lower signal. It may be due to the obvious infiltration of inflammatory cells in the early stage of the lesion and the less content of fibrous tissue $(13,14)$. (IV) Gd-EOB-DTPA enhanced examination showed the hepatobiliary specific lesions displayed a low signal, indicating a lack of liver cells in the lesions.

\section{Differential diagnosis}

BDA needs to be distinguished from other benign and malignant lesions of the liver: (I) hepatocellular carcinoma: the vast majority of clinical backgrounds include hepatitis and cirrhosis. Alpha fetoprotein (AFP) and abnormal thrombin levels have increased to varying degrees. Enhanced inspection shows the typical "fast-in, fast-out" enhancement method and the envelope enhancement is visible. However, BDA has no history of hepatitis and cirrhosis, and there are no obvious abnormalities in laboratory tests. Most of the enhancements are continuously enhanced without capsules $(5,15,16)$. (II) Intrahepatic cholangiocarcinoma: uneven signal, often accompanied by shrinkage of the adjacent liver capsule, atrophy of the hepatic lobes at the distal end of the lesion, and dilatation of the intrahepatic bile duct. The dynamic enhancement manifested as mild and moderate peripheral enhancement in the early stage, and progressive and concentric strengthening in the later stage $(17,18)$. (III) Metastatic tumors: having a history of malignant tumors in other parts, generally multiple, varying in size, enhanced with ring-shaped enhancement, and partially with "bull's eye sign" (3). (IV) Small hemangiomas: typical hemangiomas are easy to diagnose. T2WI is significantly higher in signal, enhances marginal nodular enhancement in the early stage, and improves concentric filling in the later stage. However, small hemangioma is often manifested as obvious uniform enhancement in the arterial phase, with equal or slightly higher signals in the portal and delayed phases, similar to the BDA enhancement method, but the characteristic T2WI "lighting sign" of the hemangioma can be distinguished from BDA (1). (V) Focal nodular hyperplasia $(\mathrm{FNH})$ : occurring in young women, the lesions are mostly $<5 \mathrm{~cm}$ and are located in the superficial part of the liver. T1WI, T2WI, and DWI signals are similar to BDA. Central scar and hepatobiliary specific phase showed high signal, different from BDA $(10,19)$.

In summary, the MRI manifestations of BDA are close to the liver capsule, small lesions, mostly single, rich blood supply, enhanced continuous enhancement (partial ring enhancement), etc. It is believed that with the rapid development of imaging technology and indepth understanding of BDA pathology, our imaging understanding and diagnostic level of BDA will also be significantly improved, which will help clinically formulate reasonable diagnosis and treatment strategies.

\section{Acknowledgments}

Funding: None.

\section{Footnote}

Conflicts of Interest: All authors have completed the ICMJE uniform disclosure form (available at http://dx.doi. org/10.21037/tcr.2020.02.19). The authors have no conflicts of interest to declare.

Ethical Statement: The authors are accountable for all aspects of the work in ensuring that questions related to the accuracy or integrity of any part of the work are appropriately investigated and resolved. The study was conducted in accordance with the Declaration of Helsinki (as revised in 2013). The study was approved by ethics board of Zhongshan Hospital, Fudan University (No. Y2018193 ) and informed consent was taken from all individual participants.

Open Access Statement: This is an Open Access article distributed in accordance with the Creative Commons Attribution-NonCommercial-NoDerivs 4.0 International License (CC BY-NC-ND 4.0), which permits the noncommercial replication and distribution of the article with the strict proviso that no changes or edits are made and the original work is properly cited (including links to both the formal publication through the relevant DOI and the license). See: https://creativecommons.org/licenses/by-nc-nd/4.0/.

\section{References}

1. Wei J, Zhang D, Yang J, et al. Intrahepatic bile duct adenoma (peribiliary gland hamartoma): a case report and review of literature. Int J Clin Exp Pathol 2015;8:5908-13.

2. Roy AK, Das NN. Pediatric intrahepatic bile duct 
adenoma - rare liver tumor: A case report. World J Gastrointest Surg 2019;11:414-21.

3. Moy AP, Arora K, Deshpande V. Albumin expression distinguishes bile duct adenomas from metastatic adenocarcinoma. Histopathology 2016;69:423-30.

4. Namikawa T, Tsuda S, Fujisawa K, et al. Intrahepatic bile duct adenoma in a patient with gastric cancer. Int Cancer Conf J 2018;8:7-11.

5. Sturm AK, Welsch T, Meissner C, et al. A case of biliary adenofibroma of the liver with malignant transformation: a morphomolecular case report and review of the literature. Surg Case Rep 2019;5:104.

6. Zhang Z, Zhang W. Fragmentation patterns of circulating cell-free DNA demonstrate biomarker potential for human cancers. Biotarget 2019. doi: 10.21037/ biotarget.2019.08.02.

7. Chen L, Xu MY, Chen F. Bile duct adenoma: a case report and literature review. World J Surg Oncol 2014;12:125.

8. Allaire GS, Rabin L, Ishak KG, et al. Bile duct adenoma. A study of 152 cases. Am J Surg Pathol 1988;12:708-15.

9. An C, Park S, Choi YJ. Diffusion-weighted MRI in intrahepatic bile duct adenoma arising from the cirrhotic liver. Korean J Radiol 2013;14:769-75.

10. Chuy JA, Garg I, Graham RP, et al. Imaging features of bile duct adenoma: case series and review of literature. Diagn Interv Radiol 2018;24:249-54.

11. Ahn JM, Paik YH, Lee JH, et al. Intrahepatic bile duct adenoma in a patient with chronic hepatitis $\mathrm{B}$ accompanied by elevation of alpha-fetoprotein. Clin Mol Hepatol 2015;21:393-7.

12. Koga F, Tanaka H, Takamatsu S, et al. A case of very large intrahepatic bile duct adenoma followed for 7 years. World J Clin Oncol 2012;3:63-6.

13. Kim YS, Rha SE, Oh SN, et al. Imaging findings of intrahepatic bile duct adenoma (peribiliary gland hamartoma): a case report and literature review. Korean J Radiol 2010;11:560-5.

14. Lendvai G, Szekerczes T, Illyes I, et al. Cholangiocarcinoma: Classification, Histopathology and Molecular Carcinogenesis. Pathol Oncol Res 2018. [Epub ahead of print].

15. Lee H, Yoon JH, Kim H, et al. False Positive Diagnosis of Hepatocellular Carcinoma in Liver Resection Patients. J Korean Med Sci 2017;32:315-20.

16. Ocker M. Proteomics - a new approach to identify targets and biomarkers for hepatocellular carcinoma? Biotarget 2019;3:6.

17. Lee KB. Histopathology of a benign bile duct lesion in the liver: Morphologic mimicker or precursor of intrahepatic cholangiocarcinoma. Clin Mol Hepatol 2016;22:400-5.

18. Eccles J, Thiesen A, Sandha G. Single-operator cholangioscopy for diagnosis of cholangioadenoma (bile duct adenoma) and its potential impact on surgical management. Endosc Int Open 2018;6:E1312-6.

19. Guzman IJ, Gold JH, Rosai J, et al. Benign hepatocellular tumors. Surgery 1977;82:495-503.
Cite this article as: Qin J, Zhou G, Sheng X, Liu B. Imaging features of intrahepatic bile duct adenoma in MRI. Transl Cancer Res 2020;9(3):1861-1866. doi: 10.21037/tcr.2020.02.19 